\title{
Biochemical and histological responses of Rattus novergicus (Wistar) infected by Echinostoma paraensei (Trematoda: Echinostomatidae)
}

\author{
Juberlan S. Garcia ${ }^{\mathrm{a}, \mathrm{b}}$, Cleber S. Hooper ${ }^{\mathrm{c}}$, Raquel O. Simões ${ }^{\mathrm{c}}$, Marcos Antônio J. dos Santos ${ }^{\mathrm{d}}$, \\ Arnaldo Maldonado Jr. ${ }^{a}$, Jairo Pinheiro ${ }^{\mathrm{e}, *}$ \\ a Fundação Oswaldo Cruz, Instituto Oswaldo Cruz, IOC, Laboratório de Biologia e Parasitologia de Mamíferos Silvestres Reservatórios, Av. Brasil, 4365 Rio de \\ Janeiro, RJ, CEP 21.045-900, Brazil \\ b Curso de Pós-Graduação em Ciências Veterinárias, Universidade Federal Rural do Rio de Janeiro, Seropédica, RJ, Brazil \\ c Departamento de Controle de Qualidade Animal, Centro de Criação de Animais de Laboratório (CECAL), Fundação Oswaldo Cruz, Rio de Janeiro, Brazil \\ d Universidade Federal Rural do Rio de Janeiro, Instituto de Biologia, Departamento de Biologia Animal, Km 7, BR465, CEP 23.890-000 Seropédica, RJ, Brazil \\ e Universidade Federal Rural do Rio de Janeiro, Instituto de Biologia, Departamento de Ciências Fisiológicas, Km 7, BR465, CEP 23.890-000 Seropédica, RJ, \\ Brazil
}

\section{A R T I C L E I N F O}

\section{Article history:}

Received 20 July 2010

Received in revised form 9 December 2010

Accepted 21 December 2010

\section{Keywords:}

Hepatic function

Aminotransferases

ALKP

Bilirubin

Globulin

Albumin

\begin{abstract}
A B S T R A C T
Tests were performed to evaluate the biochemical alterations in Rattus norvegicus after infection by the intestinal trematode Echinostoma paraensei. The rodents received 150 metacercariae each, serum samples were collected and the parasite load was quantified weekly until the fifth week of infection. The levels of aspartate aminotransferase (AST), alanine aminotransferase (ALT), alkaline phosphatase (ALKP), gamma-glutamyl transferase (GGT), bilirubin, glucose, total proteins and fractions and hepatic glycogen were determined. All the animals exposed to the metacercariae were infected in the first week and worms were recovered up to the third week after infection. The levels of AST, ALT, GGT, bilirubin and globulin rose in the first and/or second week and declined thereafter to levels near those of the control group. In contrast, the level of total proteins in the plasma fell significantly in the first week while the ALKP activity went down only in the fourth and fifth weeks in relation to the control group. There was no significant difference in the levels of albumin, glycogen and glucose. Infection by $E$. paraensei in $R$. norvegicus causes changes in the hepatic function, possibly resulting from the cholestasis produced by the partial obstruction of the bile duct by the helminths.
\end{abstract}

(c) 2011 Elsevier B.V. All rights reserved.

\section{Introduction}

The echinostomatidae are widely distributed geographically and infect a large number of wild and domestic animals, along with humans. Therefore, they are of great medical and veterinary interest (Fried and Graczyk, 2000).

Human echinostomiasis has been related to at least 16 species of intestinal trematodes. Among the countries

\footnotetext{
* Corresponding author. Tel.: +55 21 26814825; fax: +55 2126823222 .

E-mail address: jps@ufrrj.br (J. Pinheiro).
}

where these parasites are endemic are China, India, Korea, Malaysia, Philippines and Indonesia. In endemic areas, the parasite is usually focally transmitted and is associated with certain socioeconomic and cultural practices. The infection occurs from eating raw or poorly cooked snails, fish, crustaceans and amphibians in which the metacercariae are encysted (Graczyk and Fried, 1998).

In Brazil, eggs of Echinostoma sp. were reported in faeces obtained from partially mummified human bodies dated to between 600 and 1200 years ago (Sianto et al., 2005). However, no case of human infection has been reported recently, although Lutz (1924) recorded commercial animals found infected, such as pigs, chickens and 
ducks, along with dogs, wild birds and rodents, indicating significant zoonotic potential (Maldonado and Lanfredi, 2008).

Echinostoma paraensei Lie and Basch (1967) is a Brazilian species of echinostoma, described through an experimental life cycle, after finding naturally infected Biomphalaria glabrata. Further, the water rat Nectomys squamipes was indicated as its definitive natural host (Maldonado et al., 2001a). The adult worms were dispersed in the gastrointestinal tract (duodenum) of the definitive host, where it reaches sexual maturity (Nollen, 1996), after which some worms can migrate to the bile duct (Maldonado et al., 2001b).Despite the advances in knowledge of the pathologies produced by experimental infection (Toledo and Fried, 2005) little is known about the biochemical changes resulting from infection by echinostomatides.In clinical laboratory studies of humans, the enzymes used to evaluate hepatopathies are alanine aminotransferase (ALT), aspartate aminotransferase (AST), alkaline phosphatase (ALKP) and gamma-glutamyl transferase (GGT). In general, the increase of these aminotransferases levels indicates the occurrence of hepatocellular lesions and increases of ALKP and GGT indicates the occurrence of cholestasis or enzymatic induction (Andriolo and Borges, 1989).The aim of this study was to determine the histological and biochemical parameters in Rattus norvegicus (Wistar) experimentally infected by Echinostoma paraensei.

\section{Materials and methods}

\subsection{Parasite and experimental infection}

The isolate of E. paraensei used was obtained from specimens of $N$. squamipes that were naturally infected, captured in the municipality of Sumidouro, Rio de Janeiro state $\left(22^{\circ} 02^{\prime} 46^{\prime \prime} \mathrm{S}, 42^{\circ} 41^{\prime} 21^{\prime \prime} \mathrm{W}\right)$, after which the cycle was maintained in the Laboratório de Biologia e Parasitologia de Mamíferos Silvestres Reservatórios (IOC) FIOCRUZ-Rio de Janeiro (Brazil), by passages in hamsters (Mesocricetus auratus) as the definitive host and B. glabrata as the first and second intermediate hosts. The metacercariae utilized for infection were obtained from B. glabrata (Sumidouro isolate), raised in the laboratory and experimentally infected.

Adult hamsters infected with $E$. paraensei were euthanized in a $\mathrm{CO}_{2}$ chamber, according to the protocol approved by the Animal Use Ethics Committee (CEUA L-074/08). The adult worms were collected from the small intestine and transferred to Petri dishes containing a $0.9 \%$ physiological saline solution. The helminthes uterus was dissected to release the eggs, which were washed and incubated in dechlorinated water at $26^{\circ} \mathrm{C}$ for 14 days. After this period, the eggs were exposed to light under a $100-W$ incandescent lamp to stimulate hatching of the miracidia.

Two hundred B. glabrata specimens were individually infected with 10 E. paraensei miracidia each and after 26 days were exposed to artificial light to stimulate emergence of the cercariae. The cercariae were then placed in contact with other snails of the same species to encyst and obtain the metacercariae.

\subsection{Experimental design}

A total of 50 adult female rats (Wistar) were divided into two groups: the treatment group, infected individually with 150 E. paraensei metacercariae, and the control group, free of infection. Seven infected and three control animals were euthanized weekly, until the fifth week of infection, as described above. The experimental infection was made in duplicate $(n=100)$.

The blood was collected by cardiac puncture and the serum was separated by centrifuging at $1200 \times g$. For the histopathological analyses, the liver was fixed in DubosqBrasil solution for $24 \mathrm{~h}$, after which the material was transferred to $70 \%$ alcohol and preserved for future use.

To measure the glycogen levels, $1 \mathrm{~g}$ of liver tissue was collected, always from the same hepatic lobe of all the animals.

\subsection{Liver histopathology}

Fragments of livers from the necropsied animals were dehydrated in an ethanol crescent series with xylol and imbedded in paraffin. The thin section were obtained using $5 \mu \mathrm{m}$ and stained with hematoxylin-eosin (Humason, 1979). These images were obtained using Nikon Coolpix 4300.

\subsection{Biochemical determination}

The biochemical determination of laboratory hepatic function included the detection of AST EC2.6.1.1, ALT EC2.6.1.2, ALKP EC3.1.3.1, bilirubin, glucose and total and fractional proteins using an automated system at the Laboratório de Controle de Qualidade do Centro de Criação de Animais de Laboratório (CECAL - Fiocruz). A commercial diagnostic kit (Doles Reagents, Ltda., Brazil) was used to determine the activity of gamma-glutamyl transferase (GGT) EC2.3.2.2. The hepatic glycogen was extracted by the technique described by Pinheiro and Gomes (1994) and determined by the 3,5 DNS technique (Pinheiro and Amato, 1994).

\subsection{Statistical analysis}

Variance analysis (one-way ANOVA) was used to determine the differences between the groups and the Tukey-Kramer test was employed to compare the pairwise differences in means each week, using the Graph Pad Prism program (V.5.00.288, Prism Inc., USA). Values with $P<0.05$ were considered significant.

\section{Results}

All the animals exposed to the metacercariae were infected in the first week, and worms were found up to the end of the third week of infection (Table 1). The percentage of worms recovered in the first week was $63.70 \%$ of the number of metacercariae administered, a significantly higher rate than in the following weeks. The worms were progressively eliminated starting in the first week after infection, with rates of $23.1 \%$ and $13.2 \%$ in the second 
Table 1

Mean and standard deviation of adult worms (Echinostoma paraensei) loaded from Rattus norvegicus (Wistar) through 5 weeks of infection.

\begin{tabular}{lllll}
\hline Period of infection (weeks) & 1 & 2 & 3 & 4 \\
Worms loaded $(X \pm S D)$ & $59.87 \pm 22.14$ & $21.75 \pm 31.65$ & $12.37 \pm 21.12$ & 0 \\
\hline
\end{tabular}
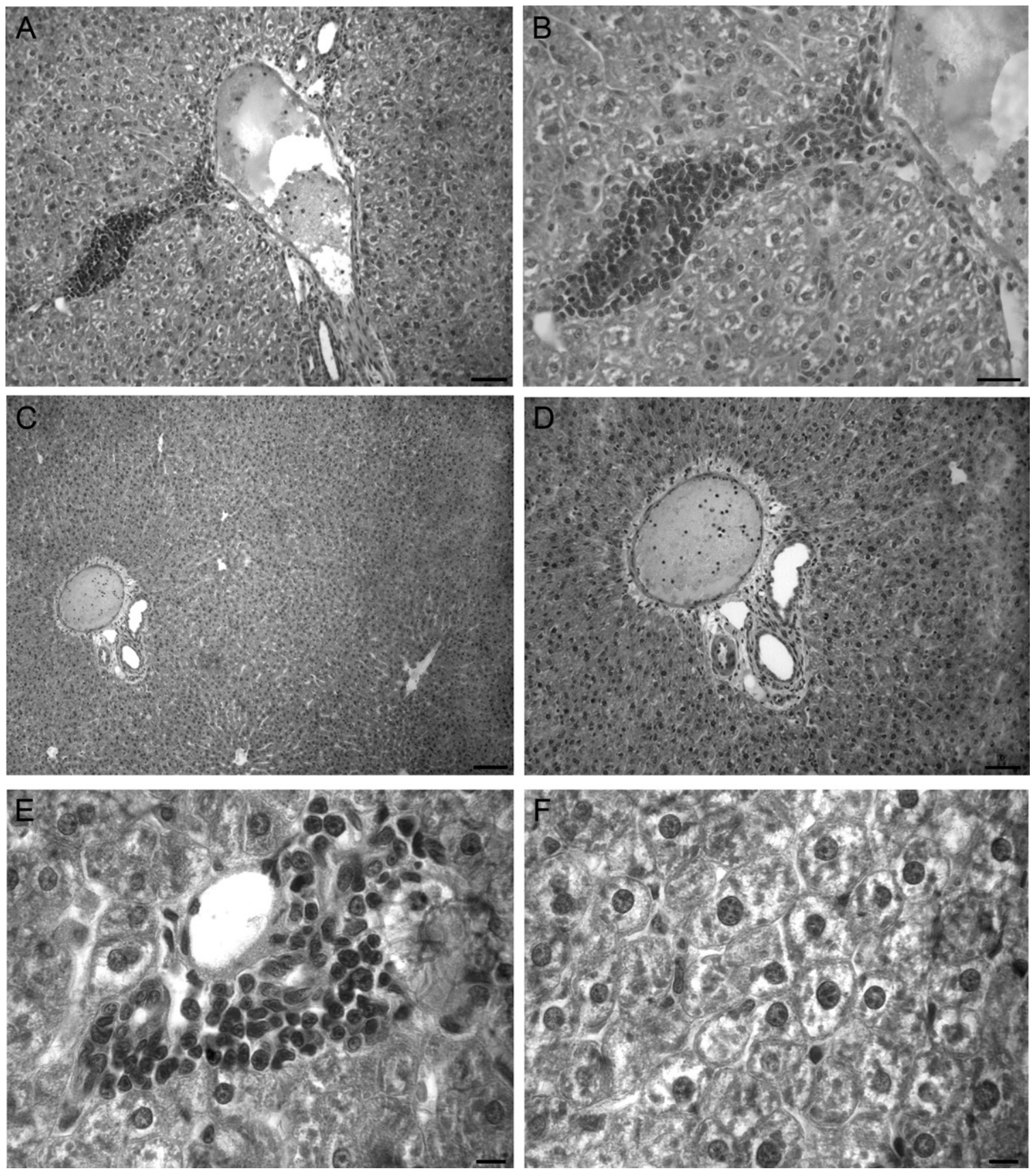

Fig. 1. Histological changes in liver Rattus norvegicus infected with Echinostoma paraensei. (a and b) The hepatocytes with infiltration of polymorphonuclear. (c and d) Portal space with estasis and inflammatory cells in the lumen of the vessel. (e and f) Hepatocytes had rounded edges, pyknotic nuclei, karyorrhexis and karyolysis, mild infiltration of polymorphonuclear and sinusoids slightly expanded. The hepatocytes were vacuolated with discrete perivascular and periportal lymphocytic infiltration expanded. Staining: HE. (a, e and f) Scale bar = $50 \mu \mathrm{m}$; (b) $\mathrm{scale}$ bar = $30 \mu \mathrm{m}$; (c) $\mathrm{scale}$ bar = $10 \mu \mathrm{m}$.

and third weeks post infection, respectively. The number of worms recovered in the first week of infection ranged from 29 to 91 .The hepatocytes had rounded edges, with signs of impaired nuclear (pyknotic nuclei, karyorrhexis and karyolysis), mild infiltration of polymorphonuclear and sinusoids slightly expanded. The hepatocytes were vacuolated with discrete perivascular and periportal lymphocytic infiltration (Fig. 1a-f). 

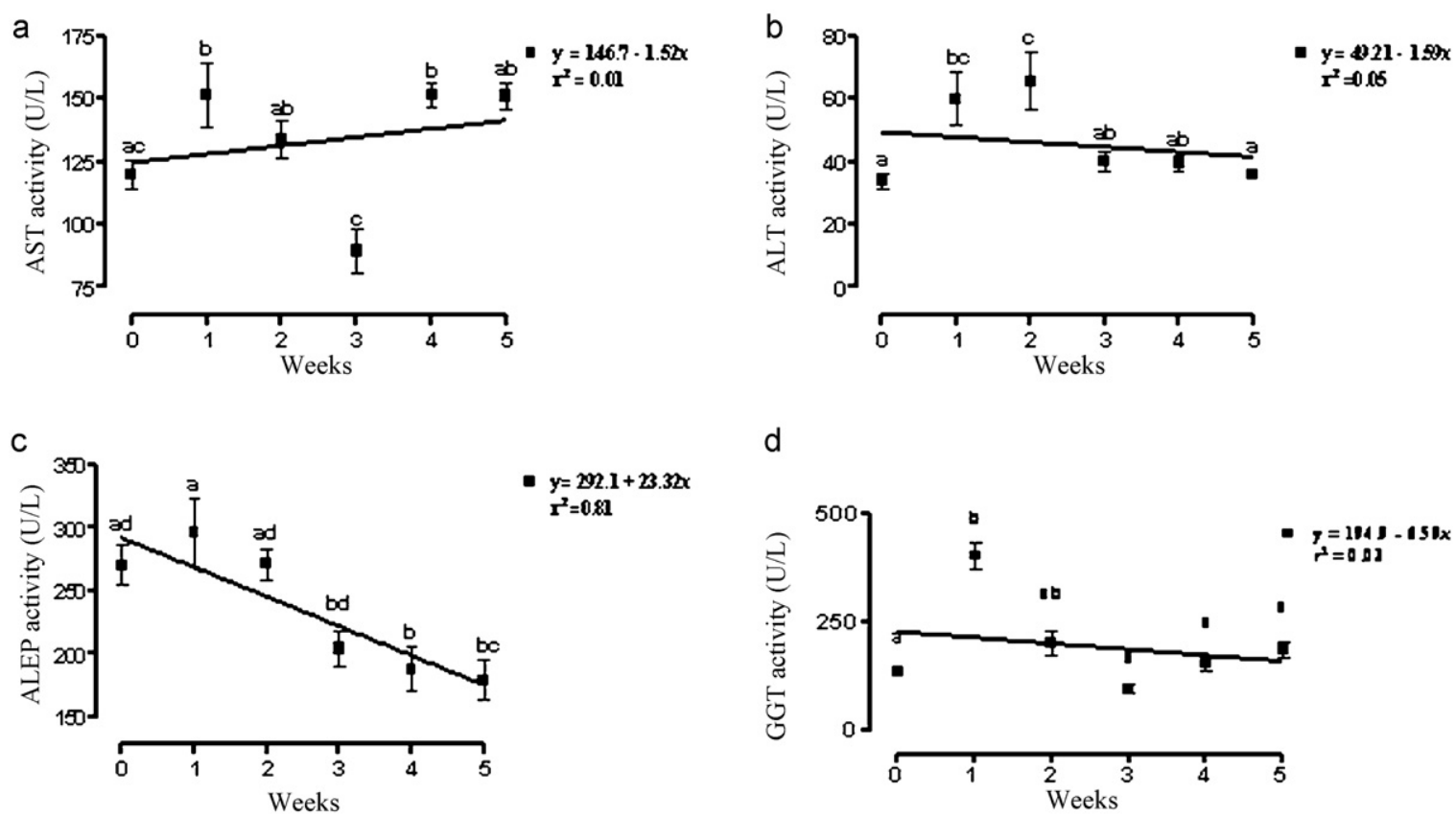

d

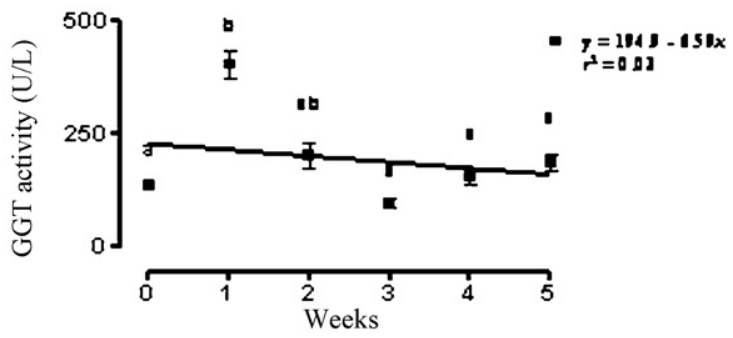

Fig. 2. Biochemical alterations in the serum of Rattus norvegicus (Wistar) experimentally infected with Echinostoma paraensei. (a) Mean values of aspartate aminotransferase (AST). (b) Mean values of alanine aminotransferases (ALT). (c) Mean values of alkaline phosphatase (ALKP). (d) Mean values of $\gamma$-glutamyl transferase (GGT). ${ }^{\mathrm{a}, \mathrm{b}, \mathrm{c}}$ Different letters indicate significant differences between values $(\alpha=5 \%)$.

The AST activity significantly increased in the first week after infection, with a level $81.97 \%$ higher than in the control group $(q=4.457 ; P<0.01)$. The activity of this enzyme was similar to that observed in the control group in the second week post infection, and then fell to levels lower than $25.3 \%$ in the third, fourth and fifth weeks in relation to the control group (Fig. 2a).

The ALT activity increased by $78.4 \%$ in the first week of infection in relation to the control group, and by $94.7 \%$ in the second week, in both cases significantly higher than in the control group. After the second week of infection, the ALT activity declined, ranging values observed in the control group (Fig. 2b).

The ALKP activity declined by $30.4 \%$ and $33.6 \%$ in relation to the control group in the fourth and fifth weeks after infection $(q=4.776$ and $q=5.270 ; P<0.01$, respectively) (Fig. 2c).

The serum levels of GGT rose significantly in the first week after infection, with an increase of $127.9 \%$ in relation to the control group. Thereafter it decreased, with percentages of $49.4 \%, 27.1 \%$ and $8.7 \%$ above the average level of the control group. However, in the fifth week post infection there was an increase of $37.9 \%$ in the GGT level (Fig. 2d).

In the first week after infection the level of total proteins in the plasma was significantly, reduced by $21.9 \%$. From the second week on the levels were greater than that observed in the control group, but the variations were not statistically significant. The level of serum globulin increased $62.5 \%$ in relation to the control group in the first week after infection. From the second week to the end of the experiment the globulin levels were near those observed in the

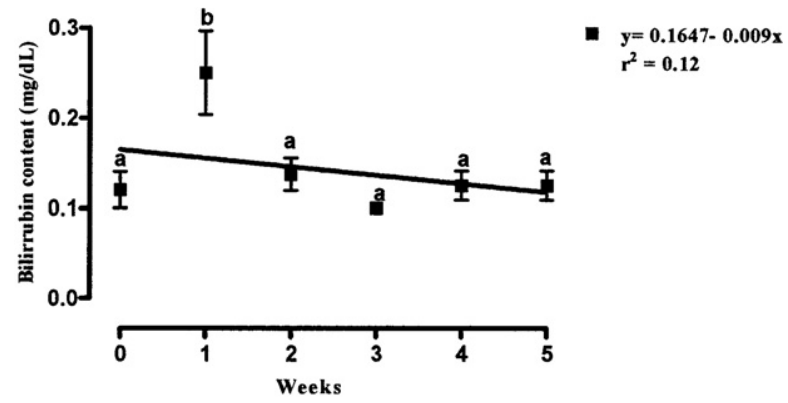

Fig. 3. Alterations in the bilirubin content in serum of Rattus norvegicus (Wistar) experimentally infected with Echinostoma paraensei. ${ }^{\mathrm{a}, \mathrm{b}}$ Different letters indicate significant differences between values ( $\alpha=5 \%$ ).

uninfected animals. There was no significant difference in the levels of albumin, glycogen and glucose between the infected and control groups (Table 2).

Finally, the bilirubin levels were significantly higher in the first week of infection $(q=5.687 ; P<0.01)$, with a rise of $108.3 \%$ in relation to the control group (Fig. 3 ).

\section{Discussion}

Experimental studies to evaluate the metabolic changes during the course of infection using the Echinostomarodent model are still incomplete and do not allow generalizations about the relation between intestinal helminths and vertebrate hosts. It has been demonstrated experimentally that Rattus norvegicus has low susceptibility to infection by Echinostoma spp., only developing the 


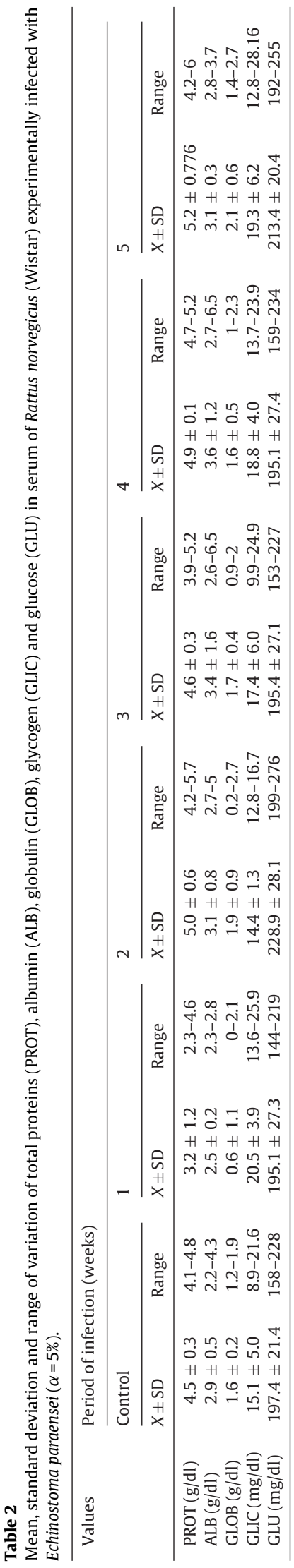

acute phase of the disease (Hansen et al., 1991; Toledo et al., 2004a, 2006).

In experimental infection of $R$. norvegicus by Echinostoma caproni, the elimination of the helminths occurred as of the fourth week of infection, and that all of them had been eliminated by the eighth week after exposure to the metacercariae (Odaibo et al., 1989). A similar characteristic was observed in experimental infection of $R$. norvegicus by Echinostoma fried, in which the parasite load remained stable until the fourth week after infection (Toledo et al., 2004b, 2006). In contrast, in the present study we observed that the helminths started to be eliminated in the second week and in the fourth no more rodents were infected by helminths. This suggests that $R$. norvegicus is less susceptible to $E$. paraensei than these two other species studied. In another study, the susceptibility of $R$. norvegicus to infection by $E$. paraensei was similar to that observed in experimental infection of the natural host $N$. squamipes (Maldonado et al., 2006), suggesting the model is suitable to study echinostomiasis.

The significant increases in the levels of ALT, AST, GGT and bilirubin in the serum of the infected animals during the initial phase of infection (first week) may be related to extrahepatic cholestasis (Burtis et al., 2006). All the worms recovered were located in the first portion of the small intestine (duodenum), near the ampulla of Vater, suggesting that the worms probably cause temporary obstruction of the bile duct. Studies by Maldonado et al. (2001b, 2005) showed that in R. norvegicus (Wistar) and in mice (Swiss Webster) infected with $E$. paraensei, the worms migrate from the small intestine to the bile duct starting in the first week of infection.

The aminotransferases ALT and AST are normally present in low concentrations in the serum (Kaplan, 1987). Although they are enzymes suggestive of hepatopathies, they are not produced solely in the hepatic tissue, but instead are distributed in other organs (Andriolo and Borges, 1989). The increase of these aminotransferases levels can be caused by various factors, due to the high sensitivity of these enzymes to a wide range of factors that can cause hepatic or extrahepatic changes. This increase of aminotransferases can also result from release of excretion-secretion products, leading to increased permeability of the hepatocyte membrane, allowing these enzymes to enter the bloodstream (Kaplan, 1987; Rej, 1971). The reduction of these enzymes to levels near those observed in the control group occurred starting in the second week of infection, in the same period the helminths were being eliminated by the host. Likewise, Dement'ev et al. (1978) also observed an increase of AST and ALT in sheep infected with 1.000 metacercariae of Dicrocoelium dendriticum, a trematode parasite of the bile duct.

The isoenzyme ALKP is present in practically all tissues of living organisms, especially in cell membranes, and it occurs in high levels in some tissues, particularly the intestinal epithelium and liver (Burtis et al., 2006). The elevation of the level of this enzyme in the serum is mainly observed when there is extrahepatic obstruction (Andriolo and Borges, 1989). In contrast, in the infection by $E$. paraensei in this study, the levels of this enzyme remained significantly lower during the infection period 
in the treatment group than in the control group. This can be due to the presence of large numbers of helminths in the intestinal mucosa, causing erosion of the villosities and injury to the intestinal epithelium, thus interfering in the production of the enzyme by the enterocytes. Mice infected by $E$. caproni presented atrophied intestinal villosities, erosion of the intestinal tissue, hyperplasia of the intestinal crypts and a reduction of ALKP activity (Weinstein and Fried, 1991; Fujino et al., 1993).

The absence of adult worms in the intestine of rats at the fourth and fifth weeks after metacercariae inoculation may explain the recovery of the parameters analyzed, except ALKP, because at this time the animals were no longer infected. Beside this, from the second week of metacercariae inoculation onward, the most of parameters of infected animals were not significantly different from those observed to uninfected (control) group, reflecting the high capacity of liver regeneration and hepatic reserve in mammals. Indeed, the data observed at fourth and fifth weeks post metacercariae inoculation already reflect the recovery of liver function after the worms' elimination. So, it is possible to infer that the results obtained in the present study, at fourth and fifth weeks, reflect a temporal recovery of the hepatic function after worm expulsion.The GGT present in the plasma originates from the hepatobiliary system, and can be used to diagnose cholestasis and obstructions of the biliary tree (Andriolo and Borges, 1989). The increase of this protease can be related directly to the obstruction of the bile duct caused by the helminth's presence. The occurrence of extrahepatic cholestasis can lead to a reduction in bilirubin extravasation into the intestine (Noguchi et al., 2002), causing an increase in the serum activity of this enzyme. This can explain the increase of this enzyme activity observed in the infection of $R$. norvegicus by $E$. paraensei.

An increase in the levels of total proteins and serum globulins can result from the response of the host's immunological system (Scott et al., 2005). In the present study, there was no significant increase in the levels of albumin. We did not observe any significant variations in the levels of glucose in the serum or glycogen in the liver of the infected animals. Manga-González et al. (2004) did no observe alterations in the levels of total proteins and glucose, and only a small increase in the levels of albumin, in lambs infected by $D$. dendriticum.

The increases in the ALT, AST, ALKP and GGT activities and bilirubin content occur at the first week of infection, when the highest worm load was observed. These results suggest that in the beginning of infection by $E$. paraensei it can cause obstruction of the bile duct and impairment of the intestinal villosities, altering the levels of ALT, AST, GGT, bilirubin and ALKP, but not causing changes in the hepatic tissue and levels of albumin, glucose and glycogen. This infection can also stimulate the activity of the immunological system, with a resulting increase in the levels of total proteins and globulin.

\section{Acknowledgments}

We gratefully acknowledge financial support from the Conselho Nacional para o Desenvolvimento Científico e
Tecnológico (CNPq) and Fundação Carlos Chagas Filho de Amparo à Pesquisa do Estado do Rio de Janeiro (FAPERJ).

\section{References}

Andriolo, A., Borges, D.R., 1989. Enzimologia clínica em doenças do fígado. Rev. Bras. Patol. Clin. 25, 95-98.

Burtis, C.A., Ashwood, E.R., Bruns, E.R. (Eds.), 2006. Tietz Textbook of Clinical Chemistry and Molecular Diagnostics. Elsevier, Amsterdam, 2448 pp.

Dement'ev, I.S., Serikbaeva, B.K., Karabaev, D.K., 1978. The effect of vitamin $\mathrm{C}$ and cobalt chloride on the pathological process in dicrocoeliasis in sheep (in Russian). Tr Kaz Nauchno-Issled. Vet. Inst. 17, 79-81.

Fried, B., Graczyk, T.K., 2000. Echinostomes as Experimental Models for Biological Research. Kluwer, Dordrecht, 273 pp.

Fujino, T., Fried, B., Tada, I., 1993. The expulsion of Echinostona trivolvis: worm kinetics and intestinal cytopathology in conventional an congenitally athymic balb/c mice. Parasitology 106, 297-304.

Graczyk, T.K., Fried, B., 1998. Echinostomatiasis: a common but forgotten food-borne disease. Am. J. Trop. Med. Hyg. 58, 501-504.

Hansen, K., Nielsen, J.W., Hindsbo, O., Christensen, N.O., 1991. Echinostoma caproni in rats: worm population dynamics and host blood eosinophilia during primary infections with 6, 25 and 50 metacercariae and resistance to secondary and superimposed infections. Parasitol. Res. 77, 686-690.

Humason, G.L., 1979. Animal Tissue Techniques, 4th ed. W.H. Freeman and Company, San Francisco, CA, USA, 661 pp.

Kaplan, M.M., 1987. In: Schiff, L., Schiff, E.R. (Eds.), Diseases of the Liver. J, L. Lippincott, Philadelphia, pp. 219-260.

Lie, K.J., Basch, P.F., 1967. The life history of Echinostoma paraensei (Trematoda: Echinostomatidae). J. Parasitol. 53, 1192-1199.

Lutz, A., 1924. Estudos sobre a evolução dos Endotrematodes brazileiros. Parte especial: 1. Echinostomidae. Mem. Inst. Oswaldo Cruz 17, 55-73.

Maldonado Jr., A., Loker, E.S., Morgan, J.A.T., Rey, L., Lanfredi, R.M., 2001a. Description of the adult worms of a new Brazilian isolate of Echinostoma paraensei (Platyhelminthes: Digenea) from its natural vertebrate host Nectomys squamipes by light and scanning electron microscopy and molecular analysis. Parasitol. Res. 87, 840-848.

Maldonado Jr., A., Coura, R., Garcia, J.S., Lanfredi, R.M., Rey, L., 2001b. Changes on Shistosoma mansoni (Digenea: Schistosomatidae) worm load in Nectomys squamipes (Rodentia: Sigmodontinae) concurrently infected with Echinostoma paraensei (Digenea: Echinostomatidae). Mem. Inst. Oswaldo Cruz 96 (Suppl. I), 193-198.

Maldonado Jr., A., Zeitone, B.K., do Carmo, Amado, L.A., Rosa, I.F., Silva, J.R.M., Lanfred, R.M., 2005. Biological variation between two Brazilian geographical isolates of Echinostoma paraensei. J. Helminthol. 79, 1-8.

Maldonado Jr., A., Gentile, R., Fernandes-Moraes, C.C., D’andrea, P.S., Lanfred, R.M., Rey, L., 2006. Helminth communities of Nectomys squamipes naturally infected by exotic trematode Schistosoma mansoni in southeastern Brazil. J. Helminthol. 80, 369-375.

Maldonado Jr., A., Lanfredi, R.M., 2008. Echinostomes in the wild. In: Fried, B., Toledo, R. (Org), The Biology of Echinostomes: From the Molecule to the Community, vol. 6. Springer, pp. 129-145.

Manga-González, M.Y., Ferreras, M.C., Campo, R., González-Lanza, C., Pérez, V., García-Marín, J.F., 2004. Hepatic marker enzymes, biochemical parameters and pathological effects in lambs experimentally infected with Dicrocoelium dendriticum (Digenea). Parasitol. Res. 93, 344-355.

Noguchi, A., Reis, J.M.C., Dias, C.S., Epaminondas, W.A., Azevedo, P.S.R., Brito, M.V.H., 2002. Serum levels of aminotransferases, bilirubins and gama-glutamyltranspeptidase after copaiba oil administration in rats. Acta Cir. Bras. 17, 1-8.

Nollen, P.M., 1996. Mating behaviour of Echinostoma caproni and E. paraensei in concurrent infections in mice. J. Helminthol. 70, 133-136.

Odaibo, A.A., Christensen, N.O., Ukoli, F.M.A., 1989. Further studies on the population regulation in Echinostoma caproni in NMRI mice. Proc. Helminthol. Soc. Washington 56, 192-198.

Pinheiro, J., Gomes, E.M., 1994. A method for glycogen determination in molluscs. Arq. Bras. Biol. Tecnol. 37, 569-576.

Pinheiro, J., Amato, S.B., 1994. Eurytrema coelomaticum (Digenea: Dicrocoeliidae): the effect of infection on carbohydrate contents of its intermediate snail host, Bradybaena similaris (Gastropoda, Xanthonychidae). Mem. Inst. Oswaldo Cruz 89, 407-410.

Rej, R., 1971. Clin. Chem. 24, 1971-1979.

Scott, P.R., Sargison, N.D., Macrae, A., Rhind, S.R., 2005. An outbreak of subacute fasciolosis in Soay sheep: ultrasonographic biochemical and histological studies. Vet. J. 170, 325-331. 
Sianto, L., Reinhard, K.J., Chame, M., Chaves, S., Mendonça, S., Gonçalves, M.L., Fernandes, A., Ferreira, L.F., Araújo, A., 2005. The finding of Echinostoma (Trematoda: Digenea) and hookworm eggs in coprolites collected from a Brazilian mummified body dated 600-1,200 years before present. J. Parasitol. 91 (4), 972-975.

Toledo, R., Espert, A., Carpena, I., Muñoz-Antoli, C., Fried, B., Esteban, J.G., 2004a. The comparative development of Echinostoma caproni (Trematoda: Echinostomatidae) adults in experimentally infected hamsters and rats. Parasitol. Res. 93, 439-444.

Toledo, R., Espert, A., Carpena, I., Muñoz-Antoli, C., Marcilla, A., Fried, B. Esteban, J.G., 2004b. Kinetics of Echinostoma caproni (Trematoda: Echi- nostomatidae) antigens in feces and serum of experimentally infected hamsters and rats. J. Parasitol. 90, 752-758.

Toledo, R., Fried, B., 2005. Echinostomes as experimental models in adult parasite-vertebrate host interactions. Trends Parasitol. 21, 251-254.

Toledo, R., Carpena, I., Espert, A., Sotillo, J., Muñoz-Antoli, C., Esteban, J.G. 2006. A quantitative approach to the experimental transmission success of Echinostoma friedi (Trematoda: Echinostomatidae) in rats. J. Parasitol. 92, 16-20.

Weinstein, M.S., Fried, B., 1991. The expulsion of Echinostoma trivolvis and retention of Echinostoma caproni in the ICR mouse: pathological effects. Int. J. Parasitol. 21, 255-257. 